\title{
Overexpression of abnormal spindle-like microcephaly-associated (ASPM) increases tumor aggressiveness and predicts poor outcome in patients with lung adenocarcinoma
}

\author{
Zhenxing Feng ${ }^{1, \#}$, Jiao Zhang ${ }^{3 \#}$, Yafang Zheng ${ }^{1}$, Jianchao Liu ${ }^{1}$, Tianyu Duan ${ }^{1}$, Tieshuan Tian ${ }^{1}$ \\ ${ }^{1}$ Department of Radiation Oncology, Tianjin Chest Hospital, Tianjin Cardiovascular Disease Research Institute, Tianjin, China; ${ }^{2}$ Tianjin Medical \\ University Cancer Institute and Hospital, National Clinical Research Center for Cancer, Tianjin's Clinical Research Center for Cancer, Key \\ Laboratory of Cancer Prevention and Therapy, Tianjin, China; ${ }^{3}$ The First Department of Breast Cancer, Tianjin Medical University Cancer Institute \\ and Hospital, National Clinical Research Center for Cancer, Key Laboratory of Cancer Prevention and Therapy, Tianjin's Clinical Research Center \\ for Cancer, Key Laboratory of Breast Cancer Prevention and Therapy, Tianjin Medical University, Ministry of Education, Tianjin, China \\ Contributions: (I) Conception and design: Z Feng, J Zhang; (II) Administrative support: T Tian; (III) Provision of study materials or patients: J Zhang; \\ (IV) Collection and assembly of data: Z Feng, Y Zheng; (V) Data analysis and interpretation: Z Feng, J Liu; (VI) Manuscript writing: All authors; (VII) \\ Final approval of manuscript: All authors. \\ \#These authors contributed equally to this work. \\ Correspondence to: Tieshuan Tian. Department of Radiation Oncology, Tianjin Chest Hospital, Tianjin Cardiovascular Disease Research Institute, \\ Taierzhuang South Road No.261, Tianjin 300222, China. Email: radiotherapytjch@126.com.
}

Background: Cumulative evidence points to abnormal spindle-like microcephaly-associated (ASPM) protein being overexpressed in various cancers, and the aberrant expression of ASPM has been shown to promote cancer tumorigenicity and progression. However, its role and clinical significance in lung adenocarcinoma (LUAD) remains unclear. This study aimed to determine the expression patterns of ASPM and its clinical significance in LUAD.

Methods: In total, 4 original worldwide LUAD microarray mRNA expression datasets $(\mathrm{N}=1,116)$ with clinical and follow-up annotations were downloaded from The Cancer Genome Atlas (TCGA) and the Gene Expression Omnibus (GEO) databases. The expression of ASPM protein in LUAD patients was detected by immunohistochemistry. Survival analysis and Cox regression analysis were used to examine the prognostic value of ASPM expression. Gene set enrichment analysis (GSEA) was performed to investigate the relationship between ASPM and LUAD.

Results: Dataset analyses and immunohistochemistry revealed that ASPM expression was significantly higher in the LUAD tissues compared with normal lung tissues, especially in the advanced tumor stage. Additionally, overexpression of ASPM was significantly correlated with shorter overall survival (OS) and relapse-free survival (RFS) in LUAD. Univariate and multivariate Cox regression analyses revealed that the overexpression of ASPM was a potential independent predictor of poor OS and RFS. However, ASPM overexpression was not significantly associated with predicting OS in lung squamous cell carcinoma. GSEA analysis demonstrated that ASPM was significantly enriched in the cell cycle, DNA replication, homologous recombination, RNA degradation, mismatch repair, and p53 signaling pathways.

Conclusions: These findings demonstrate the important role of ASPM in the tumorigenesis and progression of LUAD.

Keywords: Abnormal spindle-like microcephaly-associated (ASPM); lung adenocarcinoma; The Cancer Genome Atlas (TCGA); Gene Expression Omnibus (GEO); prognosis

Submitted Jul 22, 2020. Accepted for publication Dec 04, 2020.

doi: $10.21037 /$ tcr-20-2570

View this article at: http://dx.doi.org/10.21037/tcr-20-2570 


\section{Introduction}

Lung cancer is one of the most common cancers worldwide, with a high mortality rate. Lung adenocarcinoma (LUAD) is the most predominant subtype of lung cancer (1). Increasing our understanding of the biology and mechanisms of lung cancer has resulted in advancements in early detection and multimodal care. Furthermore, over the last 2 decades, there have been tremendous improvements in care for patients with advanced-stage lung cancer (1). However, the genesis and development of lung cancer involve the accumulation of multiple molecular events. Genetic alterations in lung cancer are associated with abnormal cell proliferation, inhibition of cell differentiation, and aggressiveness $(2,3)$. Identifying the aberrant expression of certain genes in tumors is essential for the identification of prognostic biomarkers and the development of novel therapies (4).

Abnormal spindle-like microcephaly-associated (ASPM) protein is involved in normal mitotic spindle function. However, defects in ASPM are associated with autosomal recessive microcephaly, which is a neurodevelopmental disease (5-7). An evolutionary mechanism has been suggested whereby ASPM regulates cortical expansion by controlling the affinity of ventricular radial glial cells for the ventricular surface (8). Increasing evidence indicates that ASPM can serve as a regulator of cancer stem cells. A recent study by Tsai's group revealed that ASPM modulates cancer stem cells to give rise to stemness properties and tumorigenic potential through its co-regulation of the classic Wnt- $\beta$-catenin signaling pathway in pancreatic ductal adenocarcinoma (9). Similarly, ASPM has been found to enhance tumor stemness and aggressiveness in glioblastoma (10), LUAD (11), gastric cancer (12-14), and prostate cancer (15).

Previous studies have also found that mRNA and protein expression levels of ASPM were significantly upregulated in glioblastoma tissues and glioblastoma cell lines $(10,16)$. Chen and colleagues (17) reported that the highest fold change in the differentially expressed genes was associated with ASPM, which were highly expressed in glioblastoma tissue. Furthermore, ASPM expression patterns from The Cancer Genome Atlas (TCGA) and the Gene Expression Omnibus (GEO) databases have shown that glioblastoma patients with high expression of ASPM have a poor prognosis. Mechanistically, ASPM promotes glioblastoma growth by regulating G1 restriction point progression and Wnt- $\beta$-catenin signaling. ASPM knockdown is reported to enhance radiosensitivity in glioblastoma cells by influencing
DNA double-strand break repair. Therefore, ASPM could be a potential target for combination therapy with radiation in glioblastoma (18). Several studies have revealed that increased expression of ASPM is significantly associated with poor outcomes in hepatocellular carcinoma (19), epithelial ovarian cancer (20), pancreatic tumor (21), prostate cancer (22), and bladder cancer (23). Therefore, ASPM could be a novel prognostic biomarker and therapeutic target. However, the role and clinical significance of ASPM in LUAD have not been established.

The present study explored the expression pattern of ASPM and its association with tumor progression and prognosis in LUAD patients using bioinformatics analysis. We present the following article in accordance with the MDAR checklist (available at http://dx.doi.org/10.21037/ tcr-20-2570).

\section{Methods}

\section{Worldwide microarray gene expression datasets}

Original worldwide microarray mRNA expression datasets and corresponding clinical information of lung cancer patients were downloaded from the GEO (https:// www. ncbi.nlm.nih.gov/geo/) and TCGA (https://portal.gdc. cancer.gov/) databases, respectively. TCGA-LUAD (515 patients) and 3 LUAD-related GEO datasets including GSE31210 (204 LUAD patients) (24), GSE30219 (85 LUAD patients) (25), and GSE72094 (442 LUAD patients) (26) were subjected to gene expression analyses and survival analysis. Preprocessing and analysis of raw data were performed using $\mathrm{R}$ software version 3.6.3. Additionally, the expression of ASPM mRNA in the TCGA-LUAD and GSE31210 datasets were $\log 2$-transformed for further analysis. Patients with a survival time shorter than 3 months were excluded, and a total of 1,116 LUAD patients were enrolled for survival analysis. A total of 502 lung squamous cell carcinoma (LUSC) samples and 49 normal samples were downloaded from the TCGA-LUSC dataset for gene expression analysis. Out of the 504 LUSC patients with a follow-up survival time longer than 3 months, 431 LUSC patients were included in the survival analysis. Patient information derived from the TCGA and GEO datasets is summarized in Table 1.

\section{Immunobistochemistry}

All patients treated with chemotherapy or radiotherapy before surgery were excluded from this study. Paired 
Table 1 Patients information from the TCGA and GEO datasets

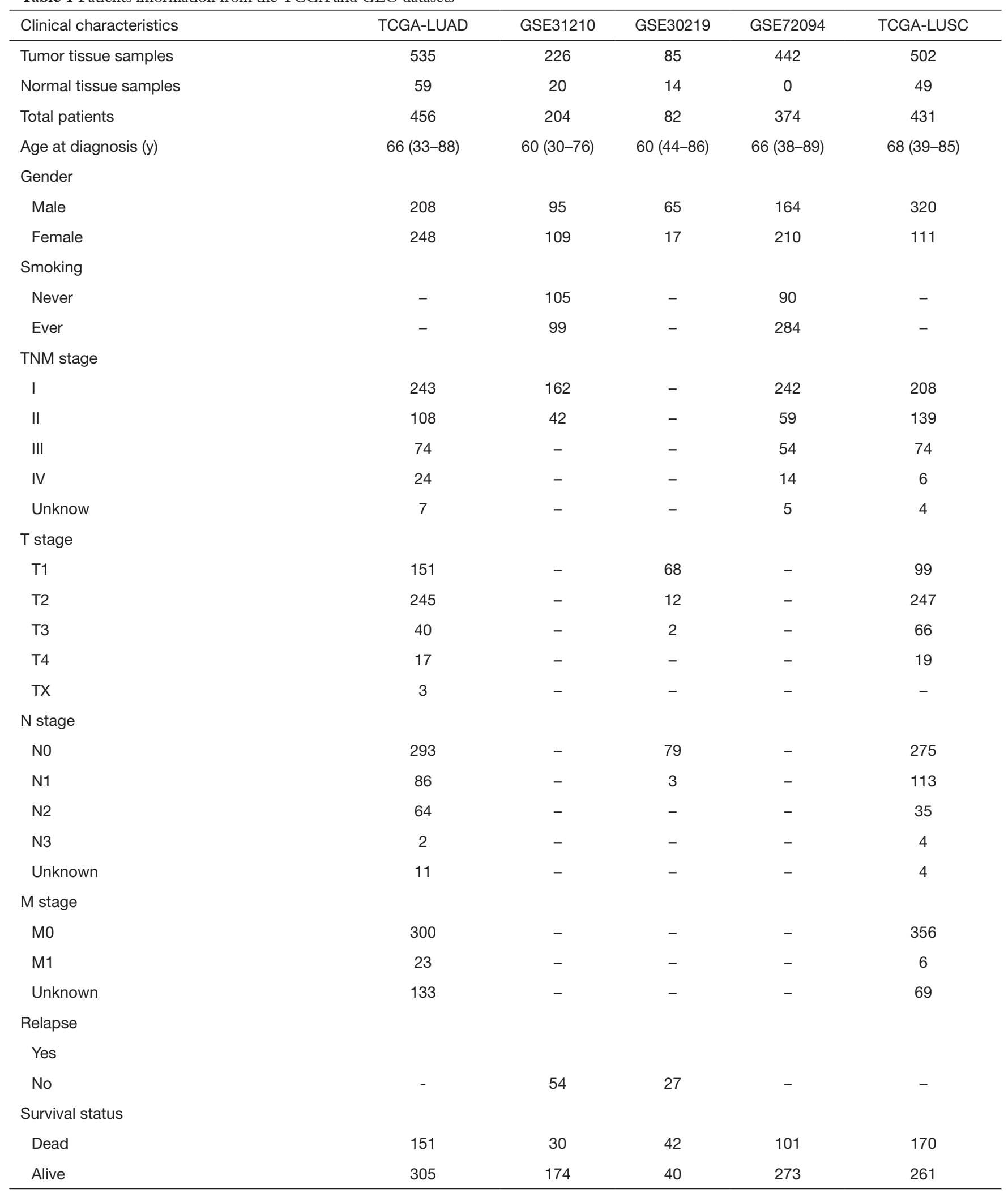

TCGA, The Cancer Genome Atlas; GEO, Gene Expression Omnibus. LUAD, lung adenocarcinoma; LUSC, lung squamous cell carcinoma. 
normal lung tissues were obtained from 32 LUAD patients who underwent surgical resection between April 2018 and February 2019. All patients were randomly selected to participate in this study. The study was conducted in accordance with the Declaration of Helsinki (as revised in 2013), and was approved by the research ethics committees of our hospital (Ek2020012). Written informed consent was obtained from all the participants. Cellular localization and expression levels of the ASPM protein in clinical LUAD tissues and paired normal lung tissues were analyzed using immunohistochemistry (IHC) as previously described $(27,28)$. The composite expression score (CES) was calculated from immunostaining patterns, yielding a range of $0-12$. The primary rabbit anti-ASPM antibody ab238106 (1:100; Abcam Corp., Cambridge, UK) and goat anti-rabbit IgG H\&L ab205718 (1:20,000; Abcam Corp., Cambridge, UK) were used for immunohistochemical analysis. The 2016 World Health Organization classification guidelines were used to perform histopathological diagnosis. The detailed clinical parameters of hospital LUAD patients are presented in Table S1.

\section{Oncomine database analysis}

The Oncomine database (https://www.oncome.org/) is a unique oncogene chip database and integrated data-mining platform offering access to published transcriptome data for various types of cancers (29). In this study, the Oncomine database was used to examine the differences in ASPM mRNA expression between tumor tissues and normal tissues in LUAD. The set parameters when filtering the data were as follows: (I) Gene: ASPM; (II) analysis type: cancer $v s$. normal analysis; (III) data type: mRNA; (IV) cancer type: lung adenocarcinoma; (V) threshold by $\mathrm{P}$ value $<1 \mathrm{e}-4$, foldchange $>2$, gene rank=top $10 \%$.

\section{Tumor immune estimation resource (TIMER) database analysis}

The Tumor Immune Estimation Resource (TIMER; https:// cistrome.shinyapps.io/timer/) web application (version 2.0) is an interactive resource for comprehensive analysis of immune infiltrates across different kinds of cancer types from TCGA dataset. Analysis of the differential expression of the ASPM gene between tumor and normal tissues in 32 different kinds of cancer types was performed in the Diff Exp module (30). The survival module was used to explore the associations between clinical outcome and ASPM gene expression.

\section{Gene set enrichment analysis (GSEA)}

The median ASPM mRNA expression level was used as the cutoff point for each of the datasets. The $535 \mathrm{LUAD}$ samples from TCGA database were divided into a highrisk ASPM group and a low-risk ASPM group. Gene set enrichment analysis (GSEA) version 3.0 (http://software. broadinstitute.org/gsea/) was used to assess the ASPM gene-associated biological pathways (31). Annotated gene datasets c2.cp.kegg.v7.0.symbols.gmt were selected as the reference gene sets. The number of permutations was set to 1,000 . Resulting pathways were selected using gene size $\geq 20$ and a false discovery rate (FDR) $q$ value $<0.05$, and ranked using normalized enrichment score (NES).

\section{Statistical analysis}

SPSS version 23.0 software (IMB, Armonk, NY, USA) and GraphPad Prism 8.02 were used to perform statistical analysis. The Shapiro-Wilk test was used to determine the normality of ASPM mRNA expression data. The MannWhitney $U$ test was used for the determination of the expression levels of ASPM between tumor tissues and normal lung tissues, and the Kruskal-Wallis test was used to compare the mean expression levels of ASPM between multiple groups. Continuous variables were grouped based on their median values. The Kaplan-Meier curve method and Cox proportional hazards regression were used to analyze overall survival (OS) and relapse-free survival (RFS). Univariate and multivariate survival analyses were used to perform further analysis. A $\mathrm{P}$ value $<0.05$ was considered to be statistically significant.

\section{Results}

\section{ASPM expression was upregulated in LUAD tissues}

By exploring the expression data from the TCGA-LUAD, GSE31210, and GSE30219 datasets, we found that ASPM mRNA expression was significantly higher in LUAD tissues compared to normal lung tissues (Figure 1A,B,C,D). ASPM transcription levels, which were validated using the Oncomine database (Figure $1 E, F, G$ ), were found to be significantly upregulated in LUAD tissues. Furthermore, results showed that ASPM mRNA expression was significantly upregulated in LUSC tissues compared with normal lung tissues based on the TCGA-LUSC dataset (Figure S1A,B). The expression of ASPM at the protein level was examined in the 32 LUAD patient samples. ASPM 
A

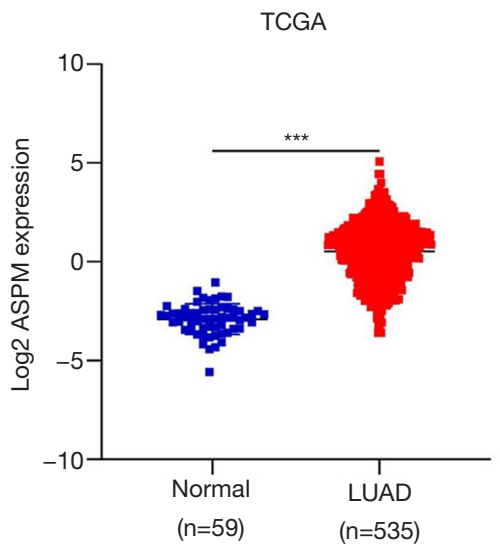

D

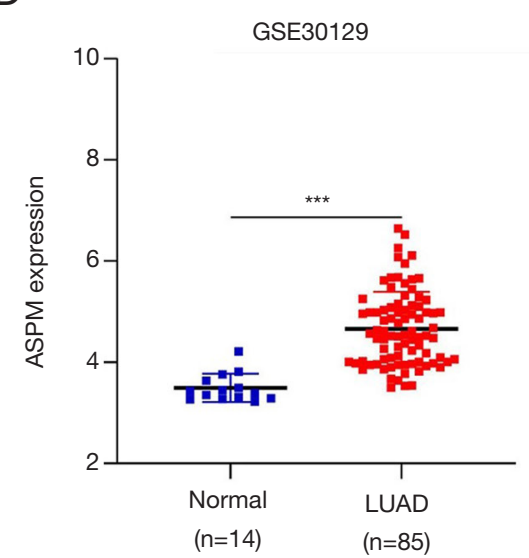

$\mathrm{F}$

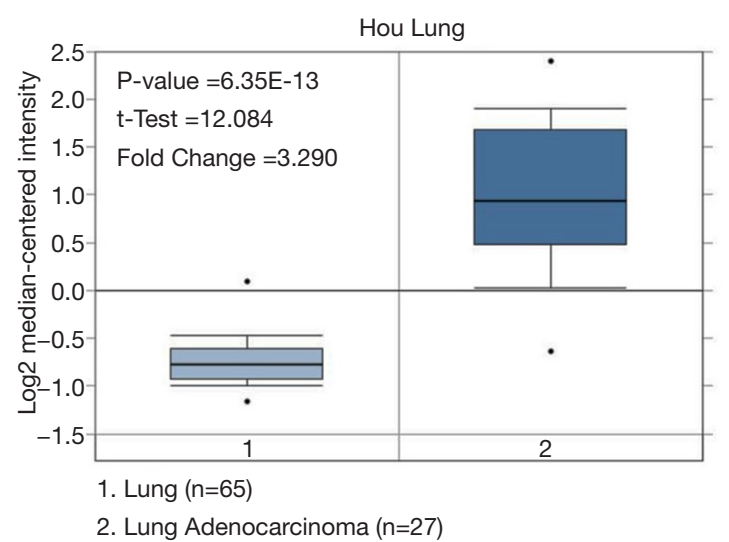

B

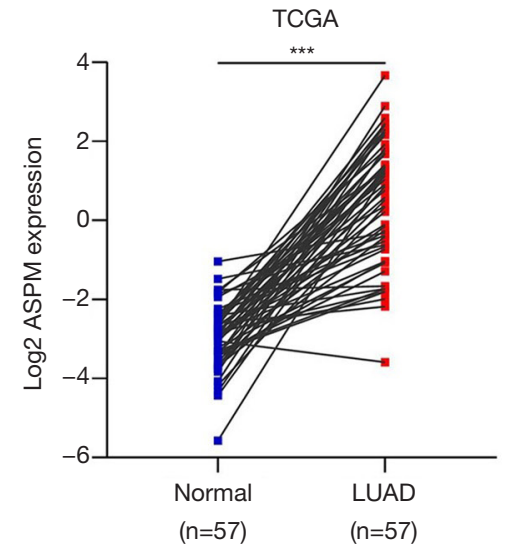

E

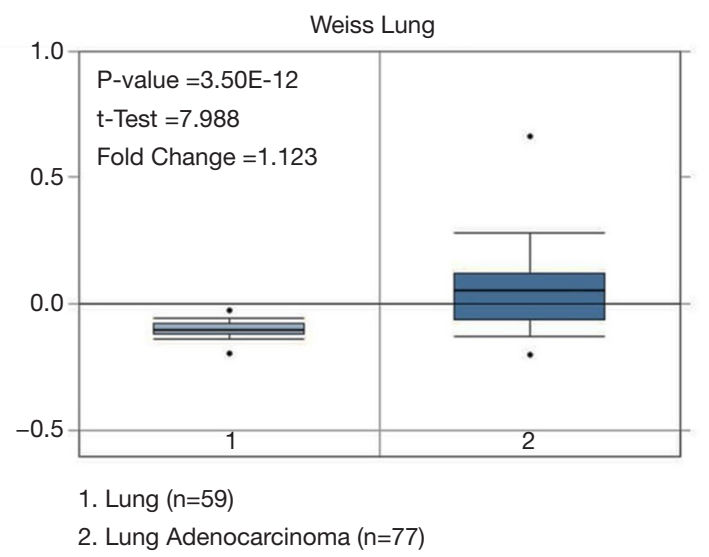

GSE31210

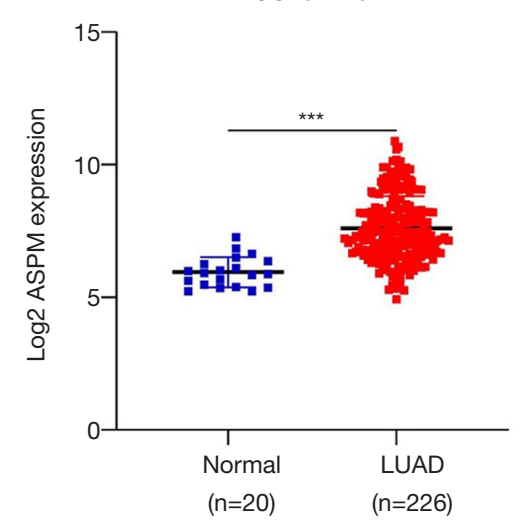

\section{G}

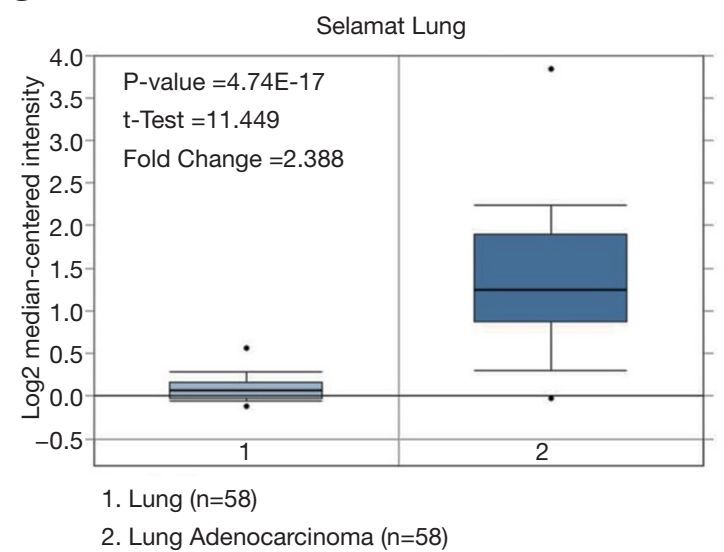

Figure 1 ASPM expression levels in LUAD based on the TCGA-LUAD and GEO datasets. (A) Expression of ASPM mRNA in LUAD and normal lung tissues in the TCGA-LUAD dataset. (B) ASPM mRNA expression in 57 LUAD tissues and paired normal lung tissues in the TCGA-LUAD dataset. (C,D) The expression difference of ASPM mRNA between LUAD and normal lung tissues in the GSE31210 and GSE30219 datasets. (E,F,G) Box plot showing ASPM mRNA levels in the Weiss Lung, Hou Lung, and Selamat Lung datasets (Oncomine). LUAD, lung adenocarcinoma; TCGA, The Cancer Genome Atlas; GEO, Gene Expression Omnibus. ${ }^{* * *} \mathrm{P}<0.001$. 
was predominantly found in the nucleus of LUAD cells and normal lung tissue cells (Figure 2A). Compared with the normal lung tissues, the expression level of ASPM protein was significantly higher in LUAD tissues (Figure 2B; CES: normal $=3.250 \pm 2.423$ vs. $\mathrm{LUAD}=6.156 \pm 2.996, \mathrm{t}=7.506$, $\mathrm{P}<0.0001)$.

\section{ASPM expression levels in different types of tumors}

The Oncomine and TIMER databases were queried for ASPM expression in different types of human cancers. In the Oncomine database, ASPM expression was upregulated in most human cancers including bladder cancer, brain and central nervous system (CNS) cancer, breast cancer, cervical cancer, colorectal cancer, gastric cancer, and lung cancer, amongst others (Figure 3A). However, ASPM expression was found to be downregulated in breast cancer and leukemia. Furthermore, in the TIMER database, expression of ASPM was found to be significantly upregulated in almost all TCGA cancer types (Figure 3B). These results indicated that ASPM is overexpressed in most human cancers.

\section{Upregulation of ASPM was an aggressive factor in LUAD}

The expression of ASPM was associated with advanced TNM stage $(\mathrm{P}=0.036)$ (Figure $4 A)$ after exploring the expression data across all patient characteristics from the TCGA-LUAD dataset. The results also showed that ASPM overexpression was significantly associated with advanced TNM stage in LUAD patients (Figure $4 B ; \mathrm{P}<0.001$ ) and early recurrence (Figure $4 C ; \mathrm{P}<0.001$ ) in the GSE31210 dataset. Similarly, further analyses of the GSE30219 dataset revealed that increased ASPM expression was significantly associated with advanced $\mathrm{T}$ stage (Figure $4 D ; \mathrm{P}<0.001$ ), $\mathrm{N}$ stage (Figure $4 E ; \mathrm{P}=0.044$ ), and early recurrence (Figure $4 F$; $\mathrm{P}<0.0001)$ in LUAD patients. These results strongly suggest that upregulated ASPM expression was significantly correlated with advanced pathological stage and early recurrence of LUAD. However, these associations were not found in LUSC patients from the TCGA-LUSC dataset (Figure S1C).

\section{Upregulated expression of ASPM predicted poor prognosis in LUAD}

Patients were separated into low- and high-expression groups based on the median mRNA expression values of ASPM. LUAD patients with high ASPM expression had significantly poorer prognosis in the TCGA-LUAD dataset (Figure $5 A ; \mathrm{n}=456, \mathrm{P}<0.001$ ). Statistical analysis among stage I-II patients showed a significant difference in OS between high- and low-expression groups in the TCGALUAD dataset (Figure $5 B ; \mathrm{n}=351, \mathrm{P}=0.002$ ). The results were validated in 3 GEO datasets. The results indicated that the OS (Figure $5 C, D ; \mathrm{n}=204, \mathrm{P}<0.01$ ) and RFS (Figure 5E,F; $\mathrm{n}=203, \mathrm{P}<0.01$ ) were significantly lower in the ASPM mRNA high expression group in the GEO31210 dataset. A similar analysis in GSE30219 with 82 primary LUAD cases showed that high expression levels of ASPM were significantly correlated with a poor OS (Figure $5 G$; $\mathrm{P}=0.007$ ) and RFS (Figure 5H, $\mathrm{P}=0.002$ ). Furthermore, high expression of ASPM mRNA was associated with a significantly shorter OS for stage I-IV patients in GSE72094 (Figure 5I; $\mathrm{n}=374, \mathrm{P}=0.001$ ). Stratification analysis indicated a significant difference in OS between high and low ASPM expression groups in early-stage patients from the GSE72094 dataset (Figure 57,K; all $\mathrm{P}<0.01)$. However, no significant association was found between ASPM expression and OS in LUSC patients from the TCGA-LUSC dataset (Figure S2).

The relationship between ASPM expression and prognosis in different cancers based on TIMER is shown in Figure S3. In this study, increased expression of ASPM was associated with poor OS in various cancers, including adrenocortical carcinoma, kidney renal clear cell carcinoma, kidney renal papillary cell carcinoma, lower-grade glioma, liver hepatocellular carcinoma, mesothelioma, pancreatic adenocarcinoma, pheochromocytoma and paraganglioma, and uterine corpus endometrial carcinoma (Figure S3A,B,C,D,E,F,G,H,I). In contrast, high expression of ASPM was associated with better OS in head and neck squamous cell carcinoma (HPV positive) and thymoma (Figure S3J,K).

\section{ASPM expression was an independent prognostic predictor for poor OS and RFS in LUAD}

Univariate and multivariate Cox regression analyses were used to evaluate the clinical prognostic value of ASPM. Univariate analysis revealed that the expression levels of ASPM mRNA ( $\mathrm{P}<0.01$ in all datasets), TNM stage $(\mathrm{P}<0.01$ in the GSE72094 and GSE31210 datasets), T stage $(\mathrm{P}<0.01$ in the TCGA-LUAD dataset), and $\mathrm{N}$ stage $(\mathrm{P}<0.01$ in TCGA-LUAD dataset) were significantly associated with OS in LUAD (Tables 2,3). Multivariate analysis showed a high level of ASPM mRNA expression $(\mathrm{P}<0.05$ in all 
A
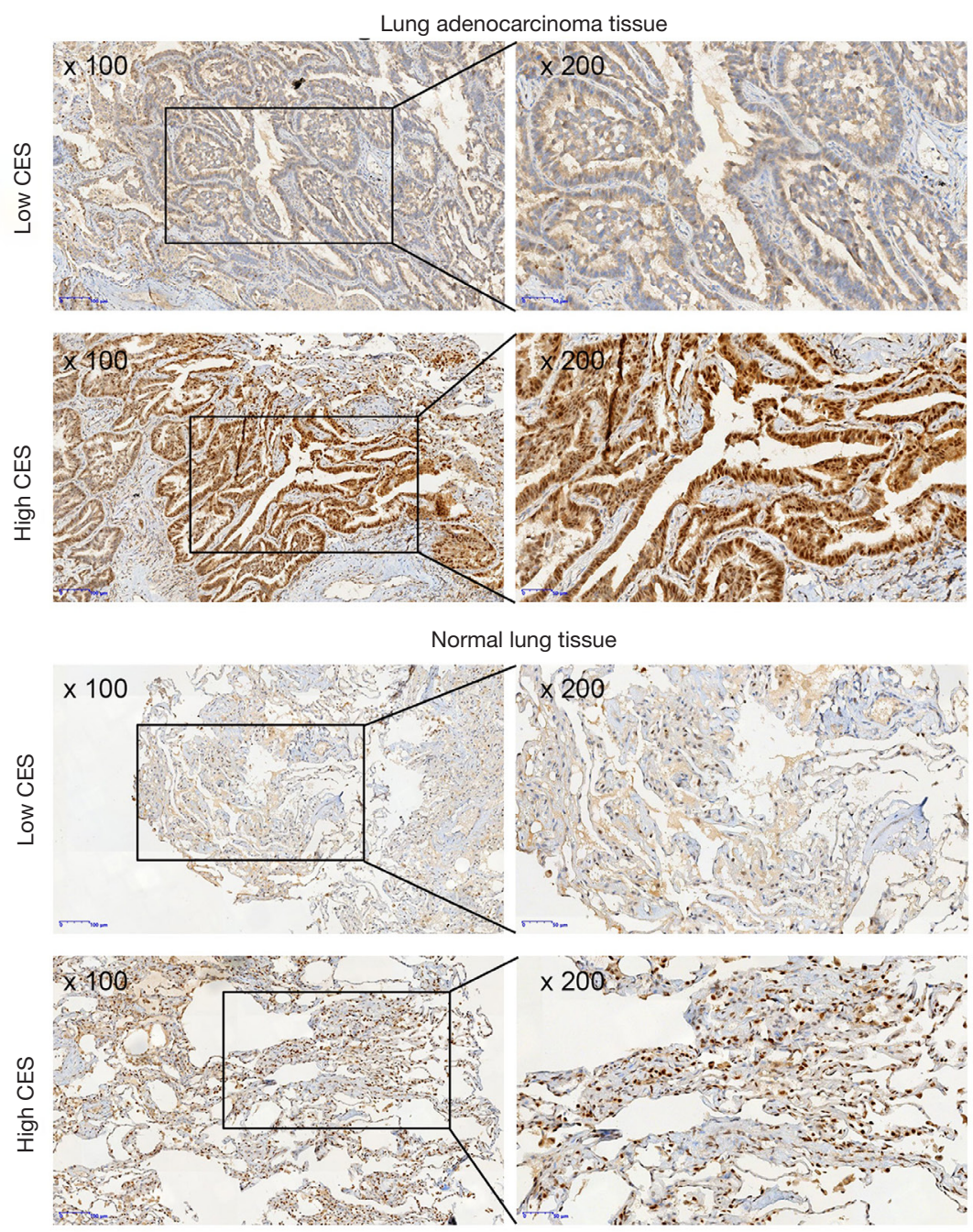

B

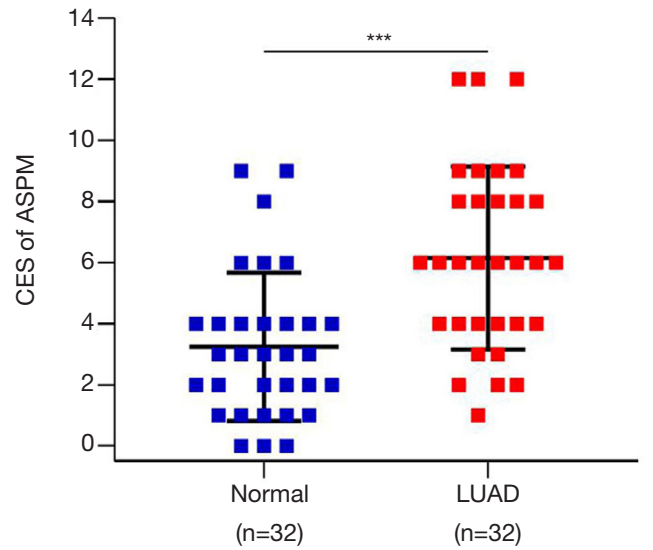

Figure 2 Immunohistochemistry analysis of LUAD tissues from 32 hospital patients. (A) Representative images of IHC staining for ASPM protein in LUAD and paired normal lung tissues (magnification, $\times 200, \times 100$ ). (B) CES of ASPM in LUAD and paired normal lung tissues. LUAD, lung adenocarcinoma; IHC, immunohistochemistry; CES, composite expression score. ${ }^{* * *} \mathrm{P}<0.001$. 

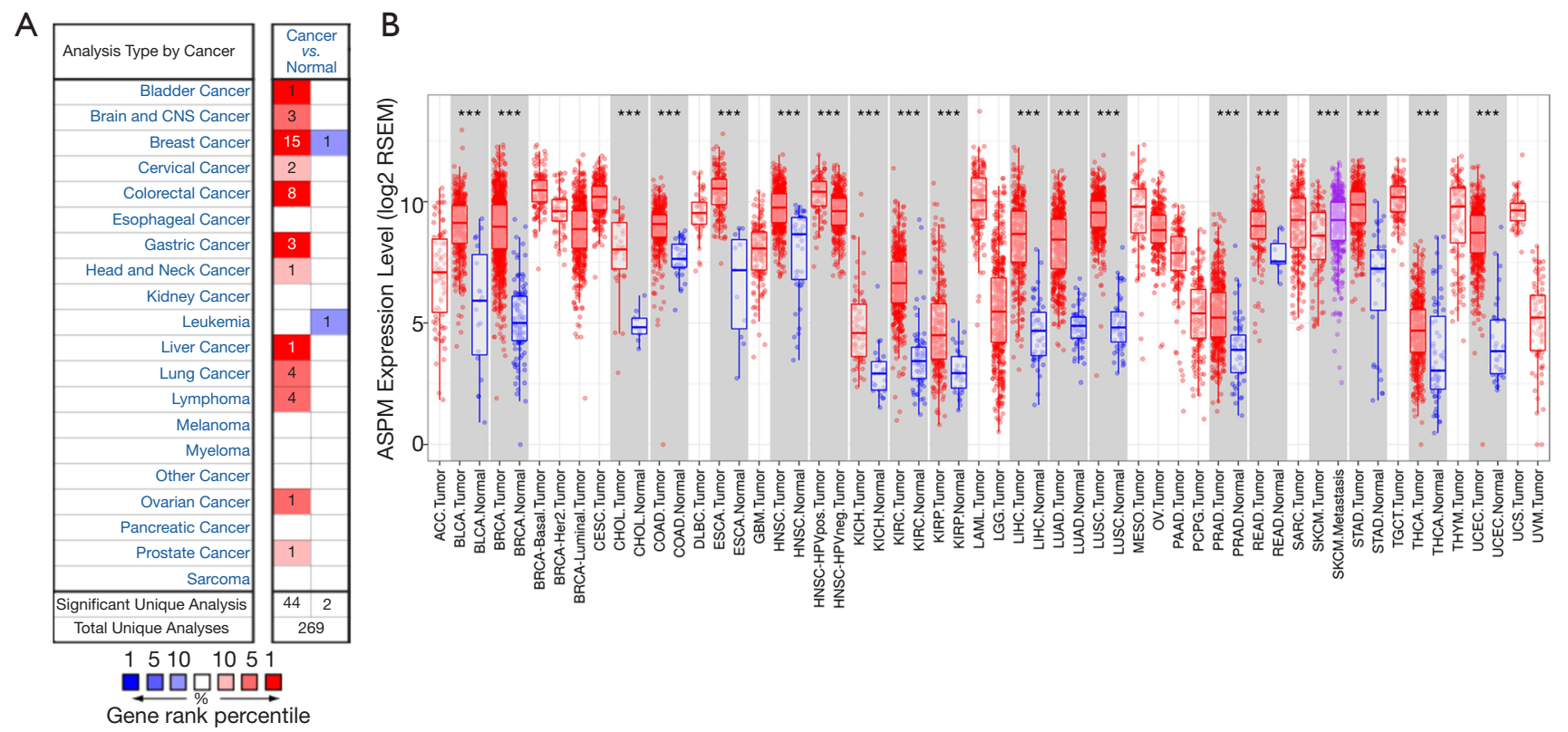

Figure 3 ASPM expression levels in different types of malignancies. (A) Upregulated or downregulated ASPM expression in different tumors compared with normal tissues in the Oncomine database. (B) ASPM expression levels in 32 kinds of cancers from TCGA database evaluated using TIMER. ${ }^{* *} \mathrm{P}<0.001$. TCGA, The Cancer Genome Atlas; TIMER, Tumor Immune Estimation Resource.

A

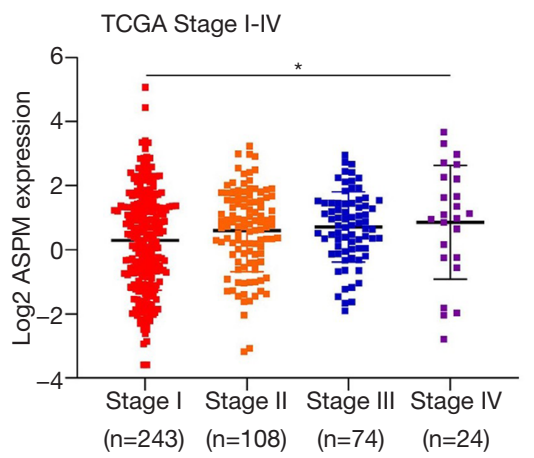

$\mathrm{D}$

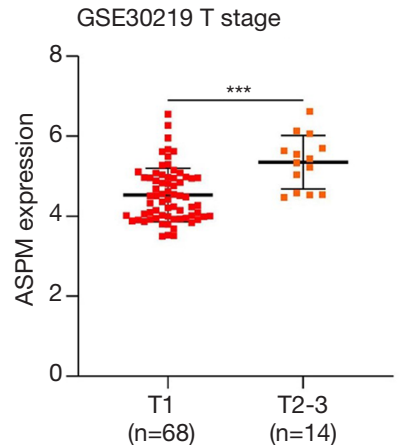

B

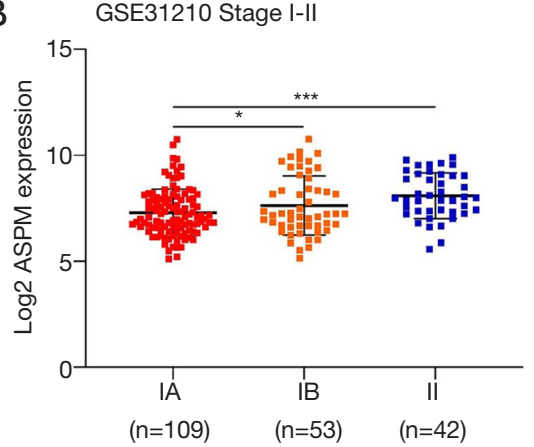

$E$

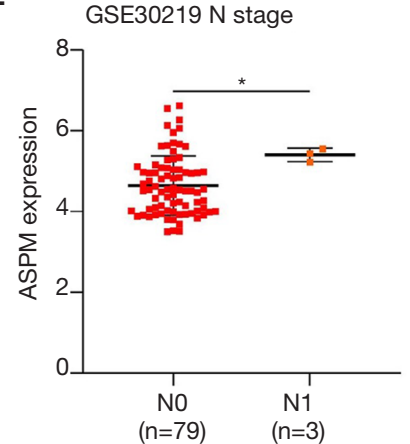

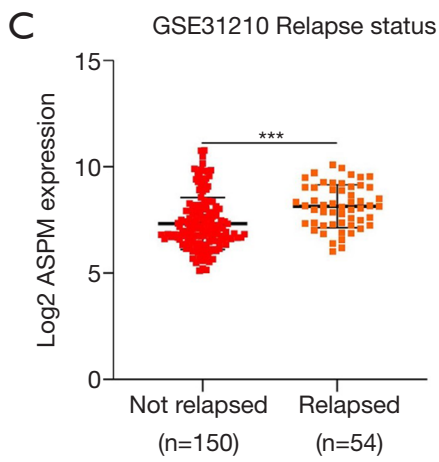

F

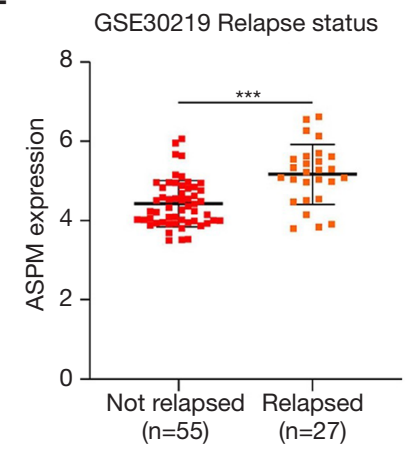

Figure 4 Correlation between ASPM expression and clinicopathological characteristics in LUAD patients. (A) The association between ASPM mRNA level with different TNM stages based on the TCGA-LUAD dataset. (B,C) The association between ASPM mRNA level with different TNM stages and relapse status based on the GSE31210 dataset. (D,E,F) The association between ASPM mRNA level with different $\mathrm{T}$ stages, $\mathrm{N}$ stages, and relapse status based on the GSE30219 dataset. LUAD, lung adenocarcinoma; TCGA, The Cancer Genome Atlas. ${ }^{*} \mathrm{P}<0.05 ;{ }^{* * *} \mathrm{P}<0.001$. 


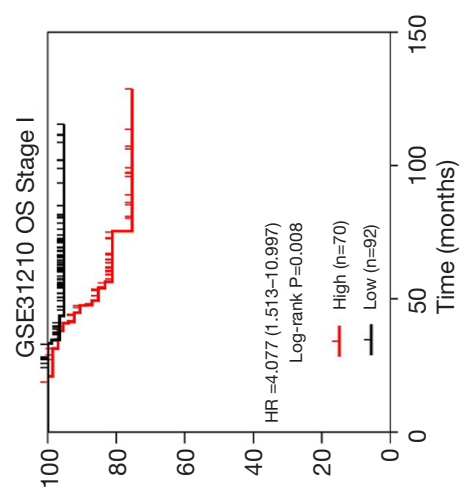

0

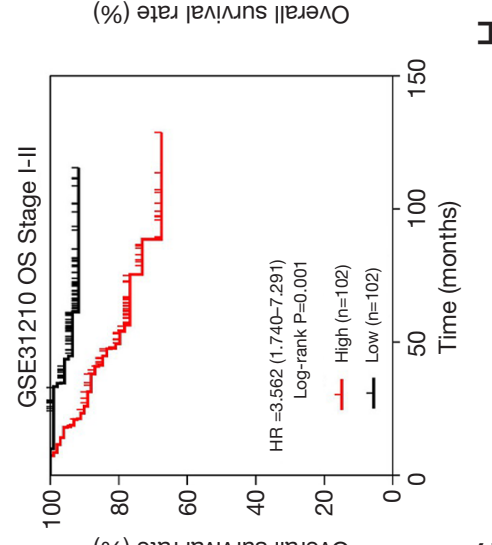

U

(\%) әреג ןел!ııns ॥еגәлО

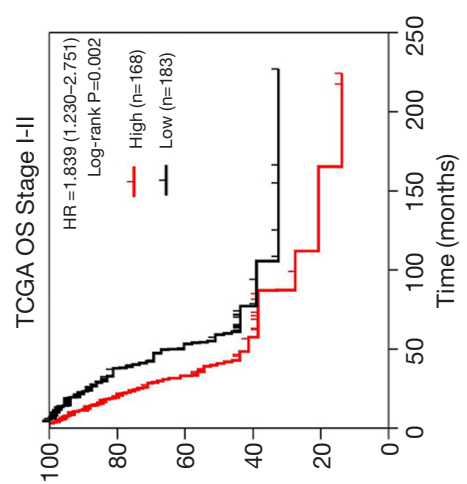

$\infty$

(\%) әреג ןел!ııns „ןеגәлО

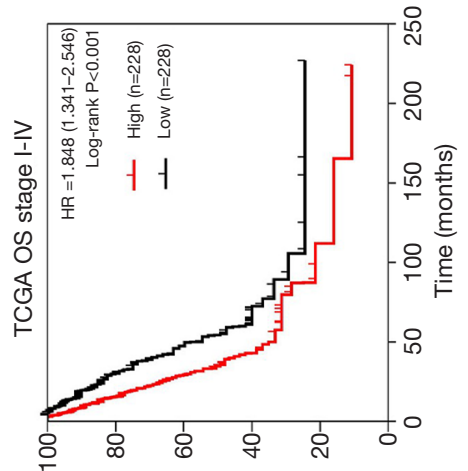

$\varangle$

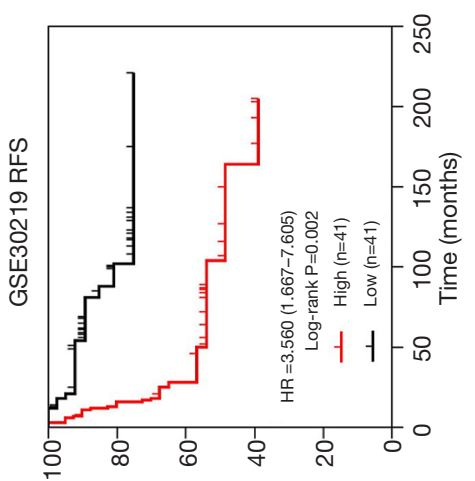

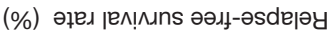

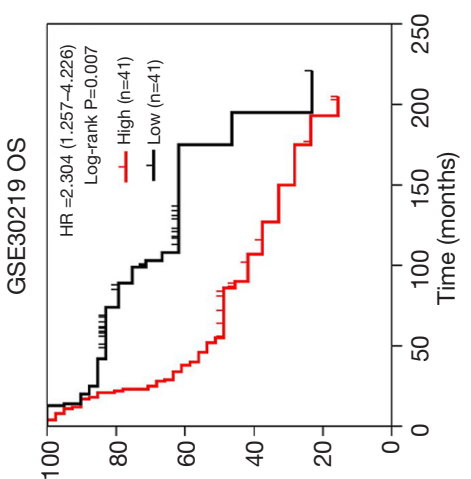

(\%) әјед ןел!ıuns „еедәло

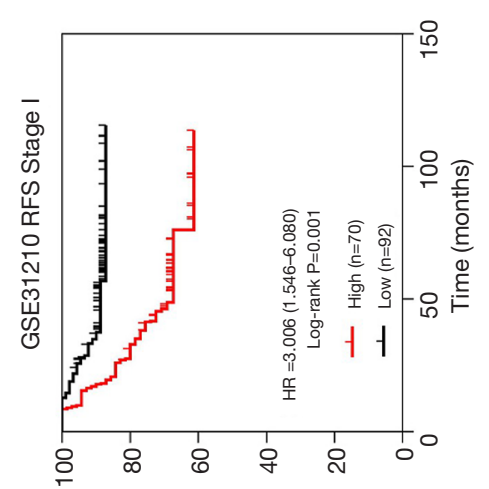

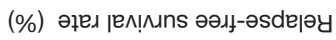

४

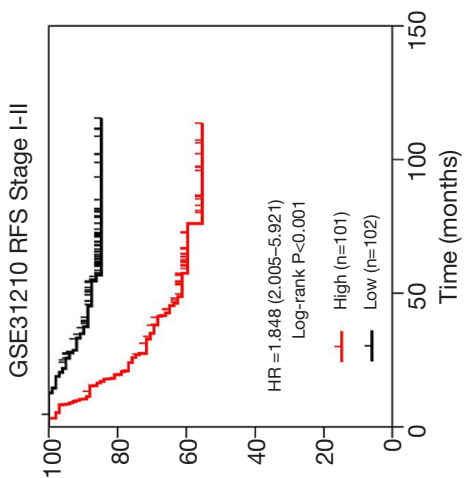

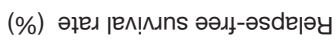

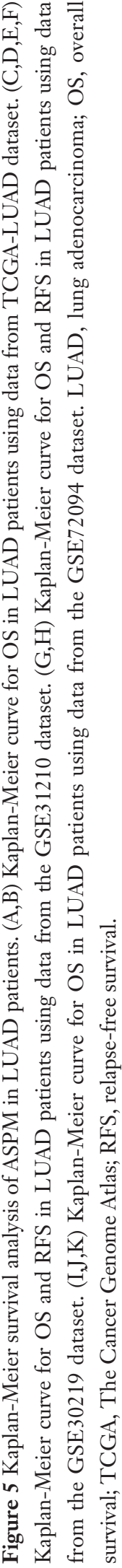


Table 2 Univariate and multivariate analysis of OS in LUAD patients in the TCGA-LUAD dataset

\begin{tabular}{|c|c|c|c|c|}
\hline Clinical characteristics & \multicolumn{2}{|c|}{ Univariate analysis } & \multicolumn{2}{|c|}{ Multivariate analysis } \\
\hline Age $(>66 / \leq 66)$ & $1.106(0.760-1.609)$ & 0.599 & $1.318(0.890-1.952)$ & 0.168 \\
\hline Gender (famale/male) & $1.038(0.716-1.506)$ & 0.844 & $1.187(0.812-1.735)$ & 0.377 \\
\hline TNM stage (IV-III/II-I) & $2.918(1.989-4.283)$ & $<0.001$ & $1.664(0.965-2.871)$ & 0.067 \\
\hline $\mathrm{N}$ stage $(1-3 / 0)$ & 2.713 (1.864-3.948) & $<0.001$ & $2.128(1.352-3.350)$ & 0.001 \\
\hline M stage $(1 / 0)$ & $1.651(0.884-3.085)$ & 0.116 & $1.011(0.484-2.112)$ & 0.976 \\
\hline ASPM (high/low) & $1.473(1.196-1.813)$ & $<0.001$ & $1.528(1.205-1.937)$ & $<0.001$ \\
\hline
\end{tabular}

OS, overall survival; LUAD, lung adenocarcinoma; TCGA, The Cancer Genome Atlas; Cl, confidence interval; HR, hazard ratio.

Table 3 Univariate and multivariate analysis of OS in LUAD patients in the GEO datasets

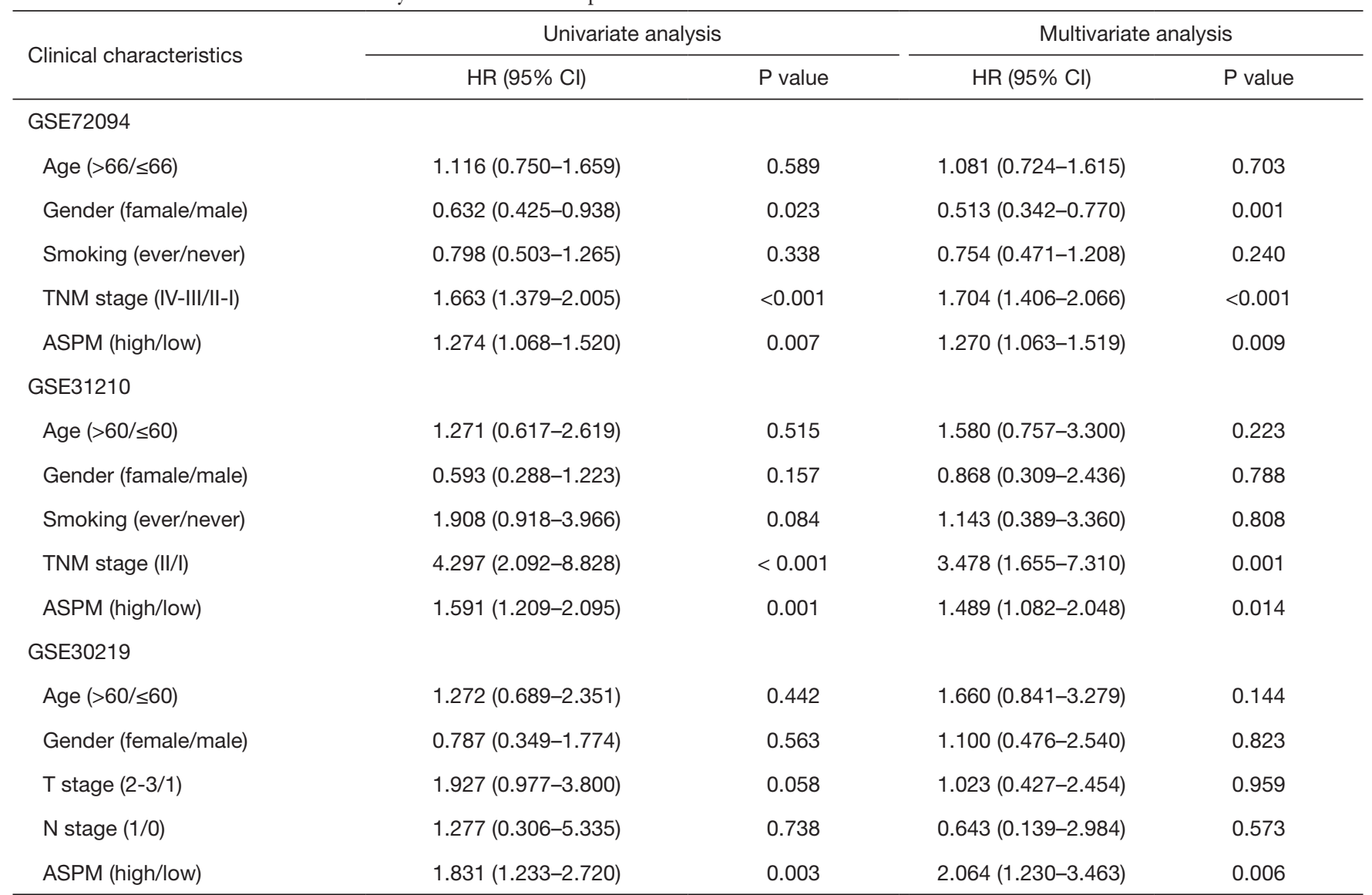

OS, overall survival; LUAD, lung adenocarcinoma; GEO, Gene Expression Omnibus; Cl, confidence interval; HR, hazard ratio.

datasets), TNM stage $(\mathrm{P}<0.05$ in the GSE72094 and GSE3 1210 datasets), and $\mathrm{N}$ stage $(\mathrm{P}<0.01$ in TCGALUAD dataset) were potential independent predictors of
OS in LUAD (Tables 2,3). The results of the univariate and multivariate Cox regression analyses revealed that the expression level of ASPM was an independent prognostic 
Table 4 Univariate and multivariate analysis of RFS in LUAD patients in the GEO datasets

\begin{tabular}{|c|c|c|c|c|}
\hline Clinical characteristics & \multicolumn{2}{|c|}{ Univariate analysis } & \multicolumn{2}{|c|}{ Multivariate analysis } \\
\hline \multicolumn{5}{|l|}{ GSE31210 } \\
\hline Age $(>60 / \leq 60)$ & $1.650(0.947-2.876)$ & 0.077 & 2.255 (1.275-3.990) & 0.005 \\
\hline Gender (famale/male) & $0.687(0.400-1.178)$ & 0.172 & $0.745(0.351-1.581)$ & 0.443 \\
\hline TNM stage (II/I) & 3.297 (1.876-5.793) & $<0.001$ & $2.998(1.671-5.378)$ & $<0.001$ \\
\hline ASPM (High/Low) & 1.553 (1.271-1.897) & $<0.001$ & 1.595 (1.268-2.006) & $<0.001$ \\
\hline \multicolumn{5}{|l|}{ GSE30219 } \\
\hline Age $(>60 / \leq 60)$ & $1.242(0.581-2.656)$ & 0.576 & $1.781(0.737-4.306)$ & 0.200 \\
\hline $\mathrm{N}$ stage $(1 / 0)$ & 3.795 (1.137-9.672) & 0.030 & $1.420(0.359-5.612)$ & 0.617 \\
\hline ASPM (high/low) & 3.152 (1.905-5.216) & $<0.001$ & $3.513(1.834-6.726)$ & $<0.001$ \\
\hline
\end{tabular}

RFS, relapse-free survival; LUAD, lung adenocarcinoma; GEO, Gene Expression Omnibus; Cl, confidence interval; HR, hazard ratio.

factor of RFS in LUAD patients after adjustment for these clinical variables (Table 4). However, no statistically significant correlation was observed between ASPM and OS in LUSC based on the TCGA-LUSC dataset (Table S2; $\mathrm{P}=0.161$ and 0.210 respectively).

\section{ASPM-associated signaling pathways in LUAD}

KEGG pathway analysis by GSEA was performed by using the TCGA-LUAD dataset to better understand the biological role of ASPM in LUAD (Figure 6). The results indicated that high ASPM expression was enriched in the cell cycle $(q<0.001)$, DNA replication $(q=0.004)$, homologous recombination $(q=0.005)$, RNA degradation $(q=0.006)$, mismatch repair $(q=0.008)$, and the $\mathrm{p} 53$ signaling pathway $(q=0.025)$. Furthermore, there was a significant negative correlation between the expression of ASPM and TP53 (Figure S4A; $\mathrm{P}<0.05$ ) based on the TCGALUAD dataset. Assessment of the relationship between ASPM expression and the TP53 mutation also revealed a significantly higher ASPM expression level in the TP53 mutation group (Figure $\mathrm{S} 4 \mathrm{~B} ; \mathrm{P}<0.0001$ ).

\section{Discussion}

Integrated bioinformatics analysis can be used to identify reliable target genes. Over the years, the application of microarray technology and bioinformatics analysis has provided LUAD microarray data that is currently available in public databases including TCGA and GEO. Using these databases, several studies have recently identified potential biomarkers including ANLN (32), integrin $\alpha 6$ (33), KRT8 (34), and DDX11 (35). These biomarkers have been shown to play potential roles in the diagnosis, staging, and treatment of LUAD.

Recent evidence suggests that the aberrant expression of ASPM promotes cancer tumorigenesis and progression $(9,15,17)$. However, the role and clinical significance of ASPM in LUAD remain unclear. Therefore, this study aimed to determine the expression pattern of ASPM and its clinical significance in LUAD. TCGA-LUAD and 3 original LUAD-related GEO datasets with a large sample size consisting of a total of $1,116 \mathrm{LUAD}$ patients, including 846 LUAD samples and 93 normal samples, were used. The expression levels of ASPM mRNA were found to be upregulated in LUAD tissue. ASPM protein expression was also found to be upregulated in LUAD tissues based on IHC results of the clinical samples. ASPM protein was found to be predominantly located in the nucleus of LUAD cells, and these findings were consistent with those reported by Wang et al. (2020) (36). In contrast, immunostaining results showed that ASPM protein was mainly localized in the cytoplasm in gastric (12) and prostate cancer (22) cells. While exploring differential intracellular distributions 
A

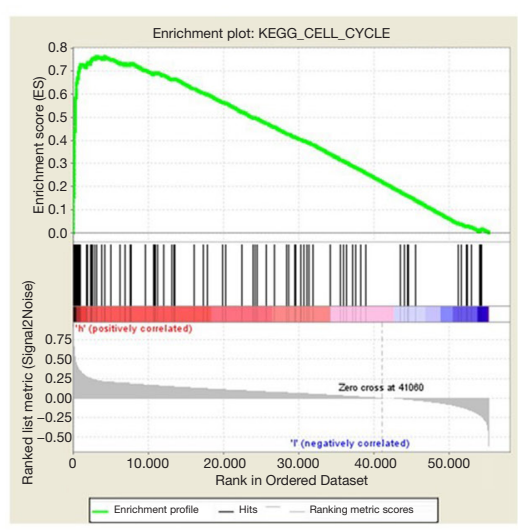

D

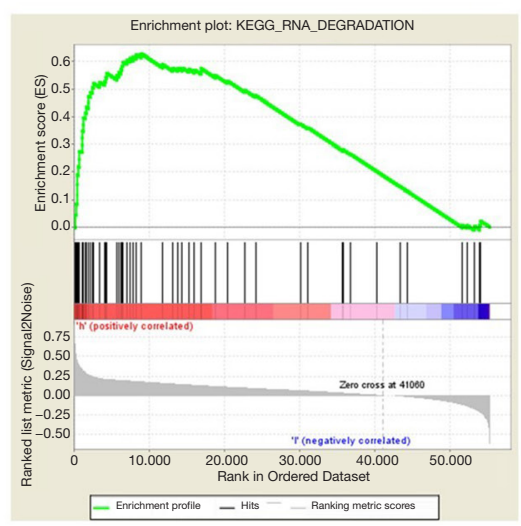

B

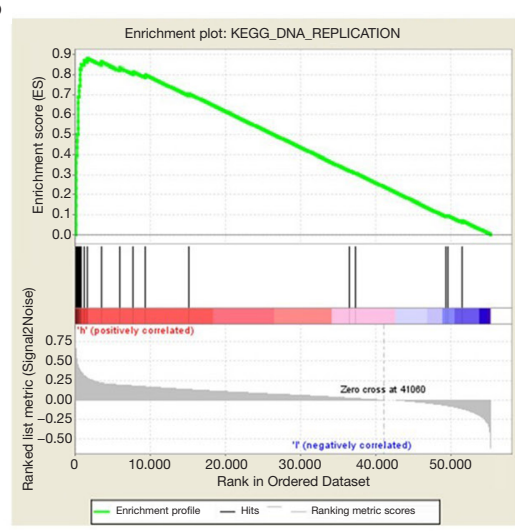

$E$

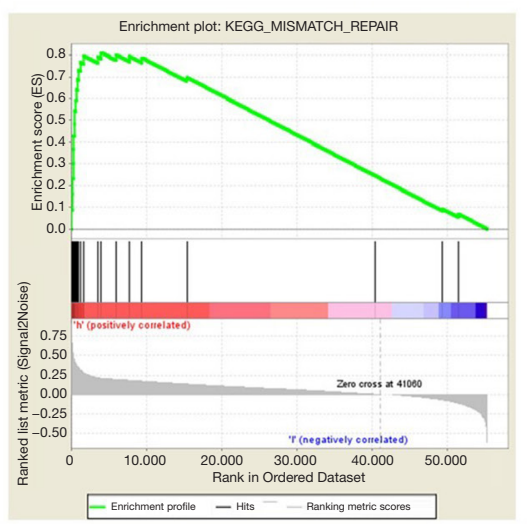

$\mathrm{C}$

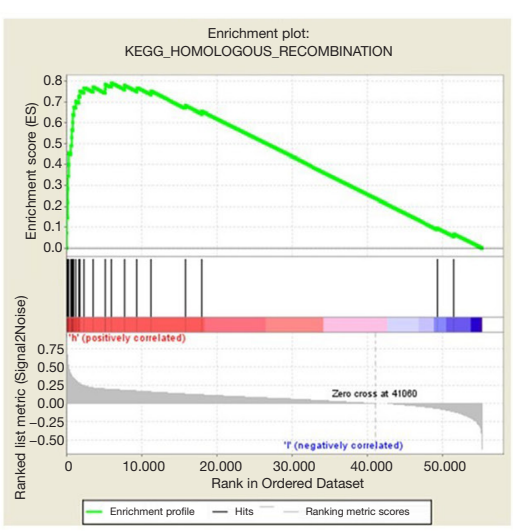

$\mathrm{F}$

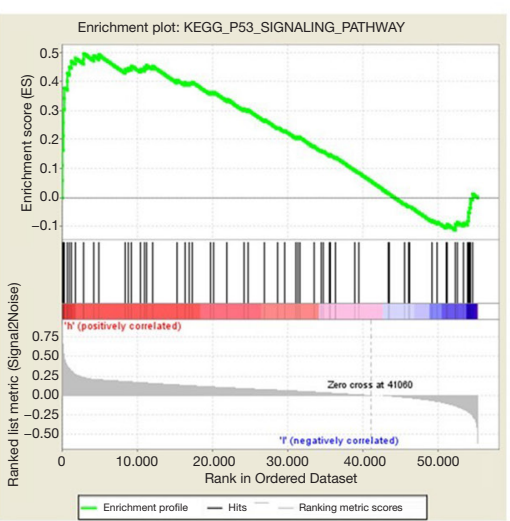

Figure 6 Gene set enrichment analysis plots. (A) Cell cycle, (B) DNA replication, (C) homologous recombination, (D) RNA degradation, (E) mismatch repair, (F) p 53 signaling pathway.

and the functions of 2 major ASPM isoforms, Hsu et al. observed that ASPM isoform I was exclusively expressed in the cytoplasm of pancreatic ductal adenocarcinoma cells, while ASPM isoform II was mainly localized in the nucleus. Brüning-Richardson et al. (20) also revealed that interphase ASPM was found both in the cytoplasm and nucleus in ovarian cancer cells. Therefore, these differences can be explained by the different ASPM isoforms, cell cycle phases, and types of cancer cells.

The findings in this study demonstrated that overexpression of ASPM was significantly associated with aggressiveness and tumor progression, early recurrence, and poor outcomes in LUAD. We further found that upregulated expression of ASPM predicted poor prognosis in patients with early-stage LUAD (Figure $5 B, C, D, E, F, \mathcal{Z}, K$ ). More importantly, overexpression of ASPM was identified as an independent prognostic factor of OS and RFS, especially in patients with early-stage LUAD. These results suggest that ASPM can be used as a biomarker for the early diagnosis of LUAD in combination with the current markers in LUAD patients. These findings also indicate the potential role of ASPM in LUAD cancer progression. Using TIMER, we further confirmed that the expression of ASPM is elevated and associated with poor outcomes in multiple cancers, including adrenocortical carcinoma, kidney renal clear cell carcinoma, and kidney renal papillary cell carcinoma, amongst others. However, ASPM overexpression in LUSC had no significant value in predicting OS based on the TCGA-LUSC dataset. A possible cause of this phenomenon may be that the genetic drivers and tumor control networks at work in LUAD versus LUSC are vastly different (37).

Consistent with these findings, previous studies have demonstrated that ASPM is significantly overexpressed in various types of cancers, including glioblastoma (17), prostate cancer (22), and bladder cancer (23). Therefore, overexpression of ASPM has been considered as a potential prognostic biomarker in many cancers. In a recent study 
of 90 Chinese patients with LUAD, ASPM expression was significantly upregulated in LUAD tissues, which was associated with poor OS (36). However, these study findings were limited by the small sample size, and thus the results concerning the association between ASPM and LUAD cannot be considered conclusive. Xu et al. (23) demonstrated that ASPM mRNA was overexpressed in bladder cancer compared with paired normal bladder mucosae. Furthermore, ASPM expression was positively associated with grade and TNM stage and short OS and RFS based on 6 bladder cancer-related datasets $(n=1,355)$ from GEO and TCGA. Another study reported that ASPM is a promising biomarker for vascular invasion, early tumor recurrence, and poor OS in hepatocellular carcinoma regardless of $\mathrm{p} 53$ mutation status and tumor stage (19). ASPM is also one of the marker genes in several independently established prognostic gene signatures of pancreatic ductal adenocarcinoma (38,39), LUAD (40), and breast cancer (41). Furthermore, integrative network analysis based on large-scale cancer genomics data from TCGA database demonstrated that ASPM is a potential target and anticancer drug repositioning candidate for precision cancer medicine in the treatment of breast and lung cancers (42).

ASPM was found to be enriched in biological pathways associated with LUAD. The 6 pathways associated with oncogenesis included the cell cycle, DNA replication, homologous recombination, RNA degradation, mismatch repair, and p53 signaling pathways. Therefore, ASPM may play a key role in oncogenesis in LUAD. The cell cycle pathway was found to be the most critical pathway based on the low $q$ value. Hsu et al. (17) reported that ASPM promotes glioblastoma growth by regulating cell cycle progression. They also revealed that the downregulation of ASPM could arrest the cell cycle of GBM cells at the G0/ G1 phase and cause a reduction in the expression of cyclin $\mathrm{E}$ and $\beta$-catenin. Similarly, Hsu et al. reported that ASPM isoform II mainly regulates cell cycle progression, especially the G1/S transition by tuning the stability of cyclin E (9). We also observed a negative correlation between the TP53 gene and expression of ASPM in TCGA-LUAD patients, suggesting a potential interaction between ASPM and TP53. Tumor suppressor gene TP53, which is mutated in most cancer cells, plays a central role in the cell cycle of cancer cells (43). Taken together, these findings suggest that ASPM may induce tumorigenesis and progression mainly through the p53 signaling pathway. However, the mechanism of ASPM in promoting LUAD remains unclear.
Therefore, further in-depth molecular studies are needed to investigate the mechanism for genetic alterations of ASPM in enhancing LUAD tumorigenesis.

Furthermore, to evaluate the potential effect of ASPM on drug responses, we analyzed the correlation between sensitivity to 84 anticancer drugs and the expression levels of ASPM using the CellMiner database (https://discover. nci.nih.gov/cellminer/home.do) (44). We discovered that sensitivity to paclitaxel, lapatinib, and salinomycin were significantly associated with ASPM, suggesting that ASPM may affect anticancer drug sensitivity in cancer cell lines (Figure S5).

There were two major limitations in this study. First, the roles of different ASPM isoforms in LUAD were not investigated due to a lack of specific antibodies for the different ASPM isoforms. Secondly, chemotherapy and radiotherapy information were not included in the datasets to explore the therapeutic significance of ASPM in LUAD.

In conclusion, integrated bioinformatics analysis of the TCGA-LUAD dataset and 3 GEO datasets confirmed mRNA and protein expression levels of ASPM to be significantly upregulated in LUAD. Overexpression of ASPM was associated with advanced TNM stage and predicted poor outcome in LUAD patients, but not in LUSC patients. Therefore, this study demonstrates that ASPM overexpression exerts significant effects on the tumorigenesis and progression of LUAD. These results, which are in agreement with those of other studies, indicate that ASPM may serve as a promising potential prognostic biomarker and therapeutic target for LUAD, and hence, provides positive prospects for future clinical transformation. However, the molecular mechanisms associated with ASPM in LUAD should be investigated, as well as the role of different ASPM isoforms in the carcinogenesis of LUAD.

\section{Acknowledgments}

Funding: This study was supported by the National Natural Science Foundation of China (grant numbers 8150111236).

\section{Footnote}

Reporting Checklist: The authors have completed the MDAR checklist. Available at http://dx.doi.org/10.21037/tcr-202570

Data Sharing Statement: Available at http://dx.doi. 
org/10.21037/tcr-20-2570

Conflicts of Interest: All authors have completed the ICMJE uniform disclosure form (available at http://dx.doi. org/10.21037/tcr-20-2570). The authors have no conflicts of interest to declare.

Ethical Statement: The authors are accountable for all aspects of the work in ensuring that questions related to the accuracy or integrity of any part of the work are appropriately investigated and resolved. The study was conducted in accordance with the Declaration of Helsinki (as revised in 2013), and was approved by the research ethics committees of the hospital (Ek2020012). Written informed consent was obtained from all the participants.

Open Access Statement: This is an Open Access article distributed in accordance with the Creative Commons Attribution-NonCommercial-NoDerivs 4.0 International License (CC BY-NC-ND 4.0), which permits the noncommercial replication and distribution of the article with the strict proviso that no changes or edits are made and the original work is properly cited (including links to both the formal publication through the relevant DOI and the license). See: https://creativecommons.org/licenses/by-nc-nd/4.0/.

\section{References}

1. Arbour KC, Riely GJ. Systemic Therapy for Locally Advanced and Metastatic Non-Small Cell Lung Cancer. JAMA 2019;322:764-74.

2. Herbst RS, Morgensztern D, Boshoff C. The biology and management of non-small cell lung cancer. Nature 2018;553:446-54.

3. Skoulidis F, Heymach JV. Co-occurring genomic alterations in non-small-cell lung cancer biology and therapy. Nat Rev Cancer 2019;19:495-509.

4. Li, Severson E, Pignon JC, et al. Comprehensive analyses of tumor immunity: implications for cancer immunotherapy. Genome Biol 2016;17:174.

5. Capecchi MR, Pozner A. ASPM regulates symmetric stem cell division by tuning Cyclin E ubiquitination. Nat Commun 2015;6:8673.

6. Tungadi EA, Ito A, Kiyomitsu T, et al. Human microcephaly ASPM protein is a spindle pole-focusing factor that functions redundantly with CDK5RAP2. J Cell Sci 2017;130:3676-84.

7. Jiang K, Rezabkova L, Hua S, et al. Microtubule minus- end regulation at spindle poles by an ASPM-katanin complex. Nat Cell Biol 2017;19:480-92.

8. Johnson MB, Sun X, Kodani A, et al. Aspm knockout ferret reveals an evolutionary mechanism governing cerebral cortical size. Nature 2018;556:370-5.

9. Hsu CC, Liao WY, Chan TS, et al. The differential distributions of ASPM isoforms and their roles in Wnt signaling, cell cycle progression, and pancreatic cancer prognosis. J Pathol 2019;249:498-508.

10. Bikeye SN, Colin C, Marie Y, et al. ASPM-associated stem cell proliferation is involved in malignant progression of gliomas and constitutes an attractive therapeutic target. Cancer Cell Int 2010;10:1.

11. Kuo WY, Wu CY, Hwu L, et al. Enhancement of tumor initiation and expression of KCNMA1, MORF4L2 and ASPM genes in the adenocarcinoma of lung xenograft after vorinostat treatment. Oncotarget 2015;6:8663-75.

12. Vange P, Bruland T, Beisvag V, et al. Genome-wide analysis of the oxyntic proliferative isthmus zone reveals ASPM as a possible gastric stem/progenitor cell marker over-expressed in cancer. J Pathol 2015;237:447-59.

13. Zhu HH, Zhuang G, Gao W. A candidate gastric stem/ progenitor cell marker revealed by genome-wide analysis. J Pathol 2016;238:3-6.

14. Wang F, Li J, Liu J, et al. Controversial role of the possible oxyntic stem cell markerASPM in gastric cancer. J Pathol 2017;241:559-61.

15. Pai VC, Hsu C, Chan T, et al. ASPM promotes prostate cancer stemness and progression by augmenting WntDvl-3- $\beta$-catenin signaling. Oncogene 2019;38:1340-53.

16. Horvath S, Zhang B, Carlson M, et al. Analysis of oncogenic signaling networks in glioblastoma identifies ASPM as a molecular target. Proc Natl Acad Sci U S A 2006;103:17402-7.

17. Chen X, Huang L, Yang Y, et al. ASPM promotes glioblastoma growth by regulating G1 restriction point progression and Wnt-beta-catenin signaling. Aging (Albany NY) 2020;12:224-41.

18. Kato TA, Okayasu R, Jeggo PA, et al. ASPM influences DNA double-strand break repair and represents a potential target for radiotherapy. Int J Radiat Biol 2011;87:1189-95.

19. Lin SY, Pan HW, Liu SH, et al. ASPM Is a Novel Marker for Vascular Invasion, Early Recurrence, and Poor Prognosis of Hepatocellular Carcinoma. Clin Cancer Res 2008;14:4814-20.

20. Brüning-Richardson A, Bond J, Alsiary R, et al. ASPM and microcephalin expression in epithelial ovarian cancer correlates with tumour grade and survival. Br J Cancer 2011;104:1602-10. 
21. Wang WY, Hsu CC, Wang TY, et al. A Gene Expression Signature of Epithelial Tubulogenesis and a Role for ASPM in Pancreatic Tumor Progression. Gastroenterology 2013;145:1110-20.

22. Xie JJ, Zhuo YJ, Zheng Y, et al. High expression of ASPM correlates with tumor progression and predicts poor outcome in patients with prostate cancer. Int Urol Nephrol 2017;49:817-23.

23. Xu Z, Zhang Q, Luh F, et al. Overexpression of the ASPM gene is associated with aggressiveness and poor outcome in bladder cancer. Oncol Lett 2019;17:1865-76.

24. Okayama H, Kohno T, Ishii Y, et al. Identification of Genes Upregulated in ALK-Positive and EGFR/KRAS/ ALK-Negative Lung Adenocarcinomas. Cancer Res 2012;72:100-11.

25. Rousseaux S, Debernardi A, Jacquiau B, et al. Ectopic Activation of Germline and Placental Genes Identifies Aggressive Metastasis-Prone Lung Cancers. Sci Transl Med 2013;5:186ra66.

26. Schabath MB, Welsh EA, Fulp WJ, et al. Differential association of STK11 and TP53 with KRAS mutationassociated gene expression, proliferation and immune surveillance in lung adenocarcinoma. Oncogene 2016;35:3209-16.

27. Wang Y, Chen C, Hua Q, et al. Downregulation of leucine-rich- $\alpha$-2-glycoprotein 1 expression is associated with the tumorigenesis of head and neck squamous cell carcinoma. Oncol Rep 2017;37:1503-10.

28. Ma Z, Liu D, Li W, et al. STYK1 promotes tumor growth and metastasis by reducing SPINT2/HAI-2 expression in non-small cell lung cancer. Cell Death Dis 2019;10:435.

29. Rhodes DR, Kalyana-Sundaram S, Mahavisno V, et al. Oncomine 3.0: Genes, Pathways, and Networks in a Collection of 18,000 Cancer Gene Expression Profiles. Neoplasia 2007;9:166-80.

30. Li T, Fan J, Wang B, et al. TIMER: A Web Server for Comprehensive Analysis of Tumor-Infiltrating Immune Cells. Cancer Res 2017;77:e108-e110.

31. Reimand J, Isserlin R, Voisin V, et al. Pathway enrichment analysis and visualization of omics data using g:Profiler, GSEA, Cytoscape and EnrichmentMap. Nat Protoc 2019; 14:482-517.

32. Long $X$, Zhou W, Wang $Y$, et al. Prognostic significance of ANLN in lung adenocarcinoma. Oncol Lett 2018;16:1835-40.

33. Shen J, Xu J, Chen B, et al. Elevated integrin $\alpha 6$ expression is involved in the occurrence and development of lung adenocarcinoma, and predicts a poor prognosis: a study based on immunohistochemical analysis and bioinformatics.
J Cancer Res Clin Oncol 2019;145:1681-93.

34. Xie L, Dang Y, Guo J, et al. High KRT8 Expression Independently Predicts Poor Prognosis for Lung Adenocarcinoma Patients. Genes (Basel) 2019;10:36.

35. Li J, Liu L, Liu X, et al. The Role of UpregulatedDDX11as A Potential Prognostic and Diagnostic Biomarker in Lung Adenocarcinoma. J Cancer 2019;10:4208-16.

36. Wang J, He Z, Duan R. Expression of ASPM in lung adenocarcinoma and Its relationship with development and prognosis. Zhongguo Fei Ai Za Zhi 2020;23:29-35.

37. Relli V, Trerotola M, Guerra E, et al. Abandoning the Notion of Non-Small Cell Lung Cancer. Trends Mol Med 2019;25:585-94.

38. Shi G, Zhang J, Lu Z, et al. A novel messenger RNA signature as a prognostic biomarker for predicting relapse in pancreatic ductal adenocarcinoma. Oncotarget 2017;8:110849-60.

39. Raman P, Maddipati R, Lim KH, et al. Pancreatic cancer survival analysis defines a signature that predicts outcome. PLoS One 2018;13:e0201751.

40. Li L, Peng M, Xue W, et al. Integrated analysis of dysregulated long non-coding RNAs/microRNAs/mRNAs in metastasis of lung adenocarcinoma. J Transl Med 2018;16:372.

41. Tang J, Lu M, Cui Q, et al. Overexpression of ASPM, CDC20, and TTK Confer a Poorer Prognosis in Breast Cancer Identified by Gene Co-expression Network Analysis. Front Oncol 2019;9:310.

42. Cheng F, Zhao J, Fooksa M, et al. A network-based drug repositioning infrastructure for precision cancer medicine through targeting significantly mutated genes in the human cancer genomes. J Am Med Inform Assn 2016;23:681-91.

43. Fischer M, Grossmann P, Padi M, et al. Integration of TP53, DREAM, MMB-FOXM1 and RB-E2F target gene analyses identifies cell cycle gene regulatory networks. Nucleic Acids Res 2016;44:6070-86.

44. Reinhold WC, Varma S, Sunshine M, et al. RNA Sequencing of the NCI-60: Integration into CellMiner and CellMiner CDB. Cancer Res 2019;79:3514-24.

(English Language Editors: C. Betlazar-Maseh and J. Gray)

Cite this article as: Feng Z, Zhang J, Zheng Y, Liu J, Duan T, Tian T. Overexpression of abnormal spindle-like microcephalyassociated (ASPM) increases tumor aggressiveness and predicts poor outcome in patients with lung adenocarcinoma. Transl Cancer Res 2021;10(2):983-997. doi: 10.21037/tcr-20-2570 


\section{Supplementary}

Table S1 CES score of 32 hospital patients with LUAD

\begin{tabular}{|c|c|c|c|c|c|c|}
\hline NO. & Age & Gender & Smoking & Stage & CES-LUAD & CES-Normal \\
\hline 1 & 60 & Male & Ever & IA & 3 & 1 \\
\hline 2 & 68 & Male & Never & IIIA & 9 & 9 \\
\hline 3 & 68 & Male & Never & IIB & 6 & 4 \\
\hline 4 & 69 & Male & Ever & IB & 2 & 1 \\
\hline 5 & 59 & Female & Ever & IIIA & 9 & 1 \\
\hline 6 & 72 & Male & Ever & IIA & 4 & 2 \\
\hline 7 & 66 & Male & Ever & IIIA & 8 & 8 \\
\hline 8 & 68 & Male & Ever & IIB & 8 & 6 \\
\hline 9 & 62 & Male & Ever & IIB & 6 & 2 \\
\hline 10 & 64 & Male & Ever & IIB & 8 & 6 \\
\hline 11 & 76 & Male & Ever & IIB & 8 & 6 \\
\hline 12 & 80 & Male & Never & IA & 2 & 0 \\
\hline 13 & 61 & Female & Ever & IIB & 4 & 2 \\
\hline 14 & 73 & Female & Ever & IIB & 4 & 3 \\
\hline 15 & 59 & Female & Never & IB & 6 & 3 \\
\hline 16 & 46 & Male & Ever & IIIB & 9 & 4 \\
\hline 17 & 72 & Female & Ever & IB & 4 & 0 \\
\hline 18 & 51 & Female & Ever & IIB & 8 & 4 \\
\hline 19 & 52 & Female & Ever & IIIA & 12 & 9 \\
\hline 20 & 62 & Female & Ever & IIIB & 12 & 4 \\
\hline 21 & 53 & Female & Ever & IA & 4 & 4 \\
\hline 22 & 63 & Female & Ever & IIIA & 9 & 3 \\
\hline 23 & 72 & Female & Ever & IA & 1 & 0 \\
\hline 24 & 72 & Female & Ever & IIIA & 12 & 4 \\
\hline 25 & 56 & Male & Ever & IA & 2 & 1 \\
\hline 26 & 59 & Female & Ever & IIB & 6 & 3 \\
\hline 27 & 59 & Male & Ever & IIIA & 6 & 3 \\
\hline 28 & 63 & Female & Never & IB & 6 & 4 \\
\hline 29 & 67 & Male & Ever & IB & 4 & 1 \\
\hline 30 & 66 & Female & Never & IB & 3 & 2 \\
\hline 31 & 62 & Male & Ever & IIIB & 6 & 2 \\
\hline 32 & 44 & Male & Ever & IIB & 6 & 2 \\
\hline
\end{tabular}

LUAD, lung adenocarcinoma; CES, composite expression score. 
A

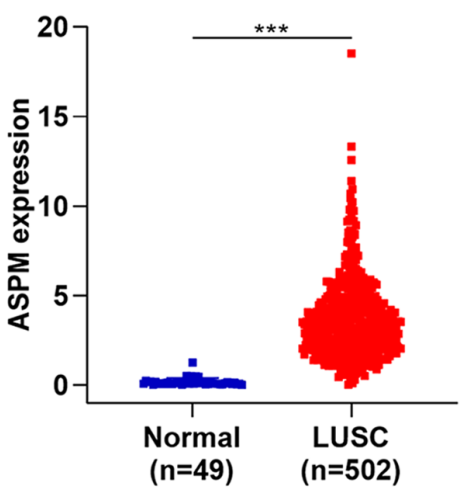

B

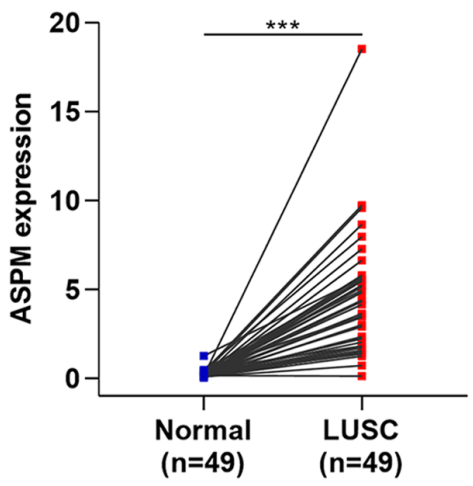

C

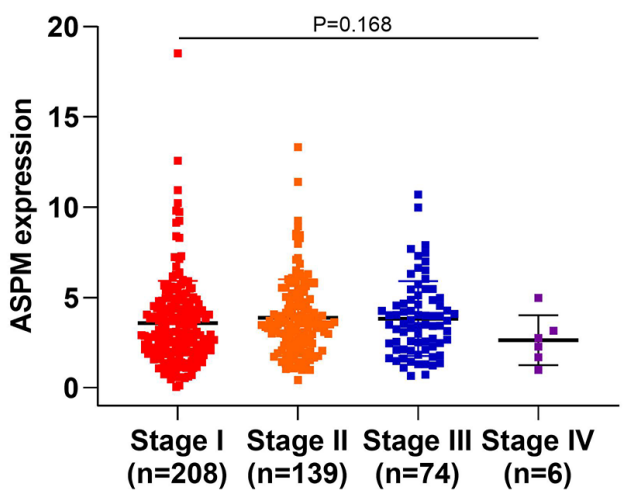

Figure S1 ASPM expression levels of LUSC in TCGA-LUSC dataset. (A) Expression of ASPM mRNA in LUSC and normal lung tissues; (B) ASPM mRNA expression in 49 LUSC tissues and paired normal lung tissues; (C) The association between ASPM mRNA level with different TNM stages in LUSC. LUSC, lung squamous cell carcinoma. ${ }^{* * *} \mathrm{P}<0.001$.

A

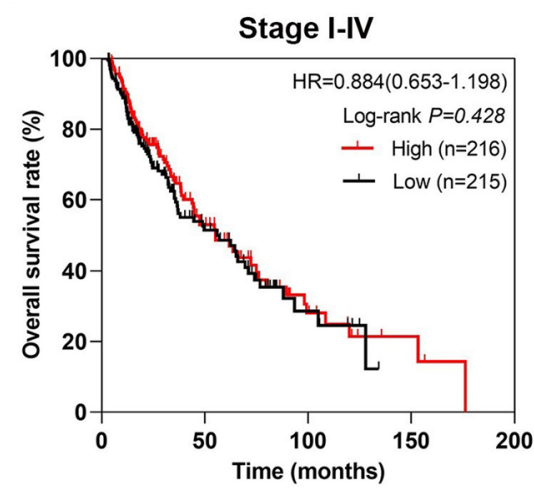

B

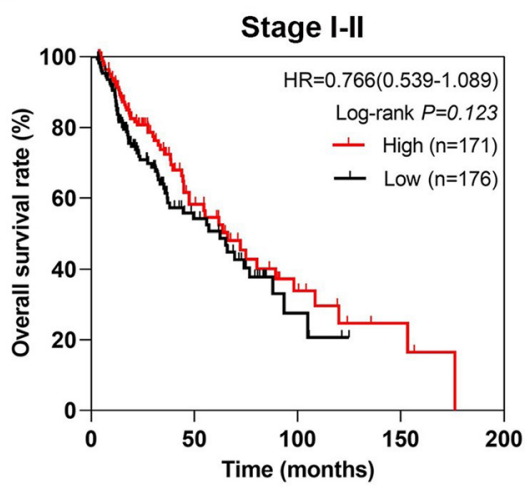

C

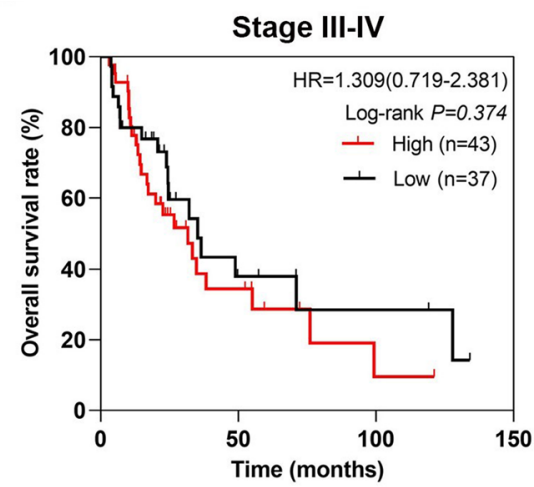

Figure S2 Kaplan-Meier survival analysis of ASPM in LUSC patients. Kaplan-Meier curve for OS in LUSC patients using data from the TCGA-LUSC dataset and based on pathological stages. LUSC, lung squamous cell carcinoma; TCGA, The Cancer Genome Atlas; OS, overall survival. 
A

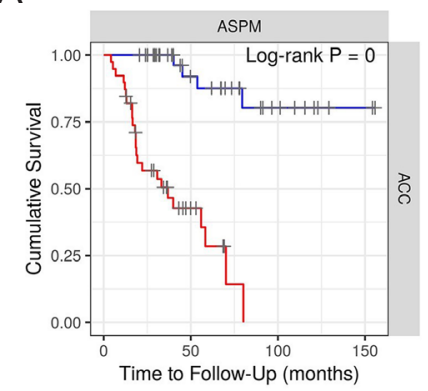

E

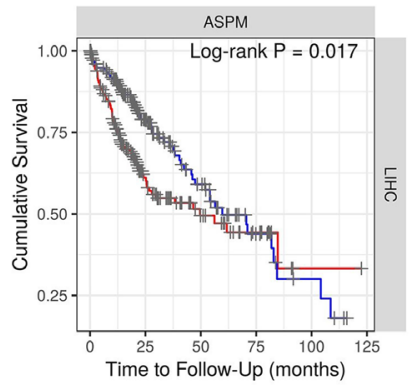

I

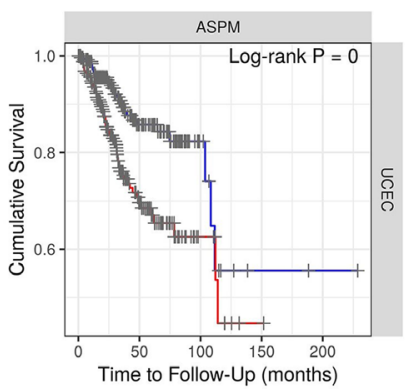

B

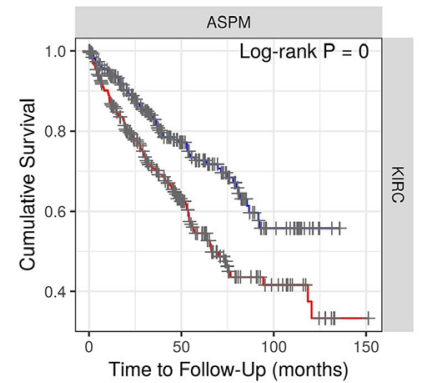

F

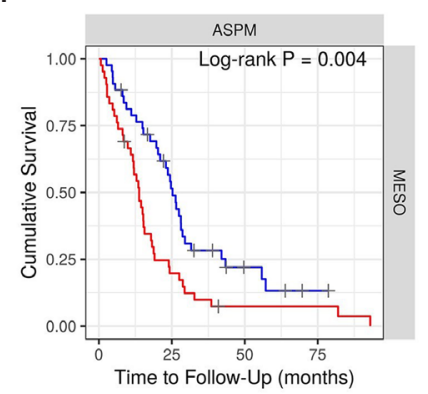

J

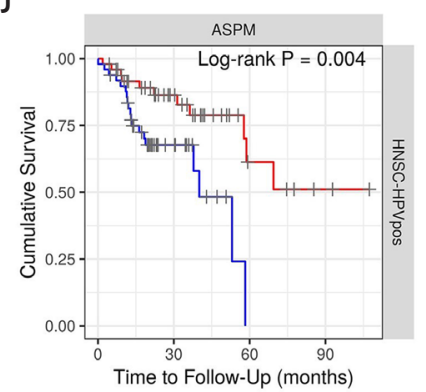

C

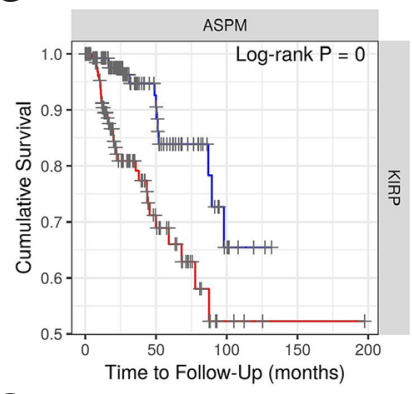

G

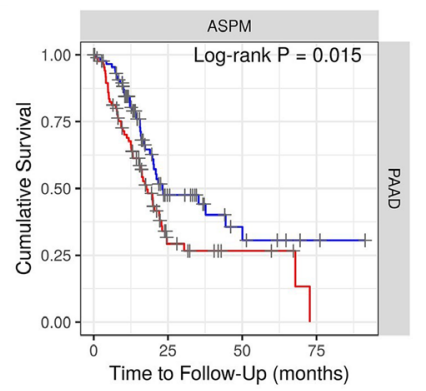

K

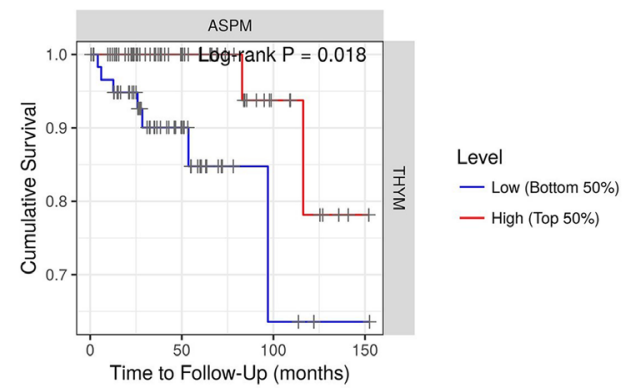

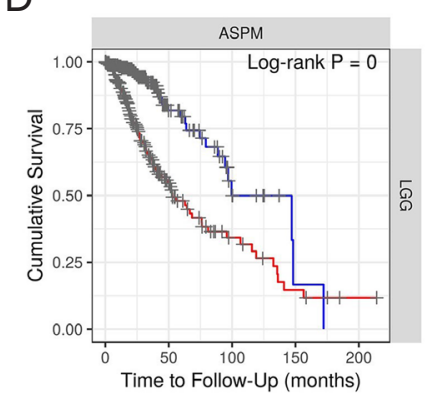

$\mathrm{H}$

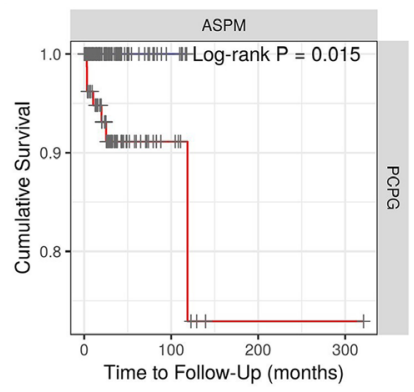

8

Figure S3 Kaplan-Meier survival analysis of ASPM in different types of cancer from TIMER. Survival curves of OS in ACC, KIRC, KIRP, LGG, LIHC, MESO, PAAD, PCPG, UCEC, HNSC-HPV pos, and THYM. TIMER, Tumor Immune Estimation Resource; OS, overall survival; ACC, adrenocortical carcinoma; KIRC, kidney renal clear cell carcinoma; KIRP, kidney renal papillary cell carcinoma; LGG, lower-grade glioma; LIHC, liver hepatocellular carcinoma; MESO, mesothelioma; PAAD, pancreatic adenocarcinoma; PCPG, pheochromocytoma, and paraganglioma; UCEC, uterine corpus endometrial carcinoma, HNSC-HPV pos, head, and neck squamous cell carcinoma-HPV positive; THYM, thymoma. 
Table S2 Univariate and multivariate analysis of OS in LUSC patients in the TCGA-LUSC dataset

\begin{tabular}{|c|c|c|c|c|}
\hline Clinical characteristics & \multicolumn{2}{|c|}{ Univariate analysis } & \multicolumn{2}{|c|}{ Multivariate analysis } \\
\hline Age $(>66 / \leq 66)$ & $1.364(0.964-1.930)$ & 0.080 & 1.467 (1.028-2.094) & 0.035 \\
\hline Gender (Famale/Male) & 0.725 (0.479-1.099) & 0.130 & $0.714(0.469-1.087)$ & 0.116 \\
\hline TNM stage (IV-III/II-I) & $1.551(1.056-2.278)$ & 0.025 & $1.221(0.703-2.121)$ & 0.478 \\
\hline$N$ stage $(1-3 / 0)$ & $1.224(0.871-1.720)$ & 0.245 & $1.168(0.792-1.720)$ & 0.433 \\
\hline M stage $(1 / 0)$ & $2.233(0.707-7.053)$ & 0.171 & $1.369(0.412-4.553)$ & 0.608 \\
\hline ASPM (High/Low) & $0.943(0.869-1.024)$ & 0.161 & $0.949(0.875-1.030)$ & 0.210 \\
\hline
\end{tabular}

OS, overall survival; LUSC, lung squamous cell carcinoma; TCGA, The Cancer Genome Atlas; Cl, confidence interval; HR, hazard ratio. 
A

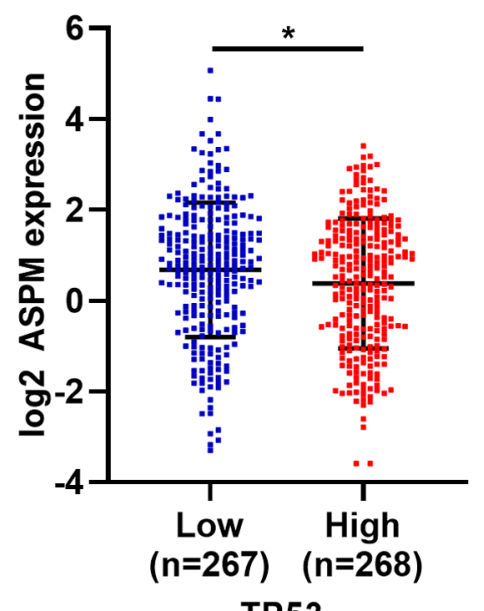

B

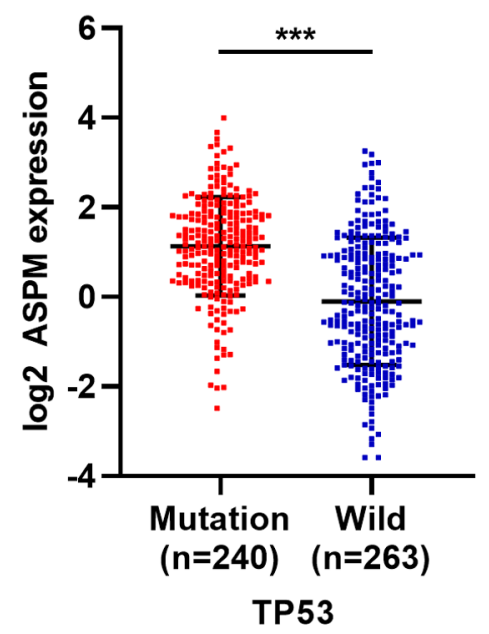

Figure S4 Association between ASPM expression and TP53 abnormality in LUAD based on TCGA dataset. (A) Comparison of ASPM mRNA expression between TP53 high group and low group. (B) Comparison of ASPM mRNA expression between TP53 mutation group and TP53 wild group. LUAD, lung adenocarcinoma; TCGA, The Cancer Genome Atlas. ${ }^{*} \mathrm{P}<0.05 ;{ }^{* * *} \mathrm{P}<0.001$.
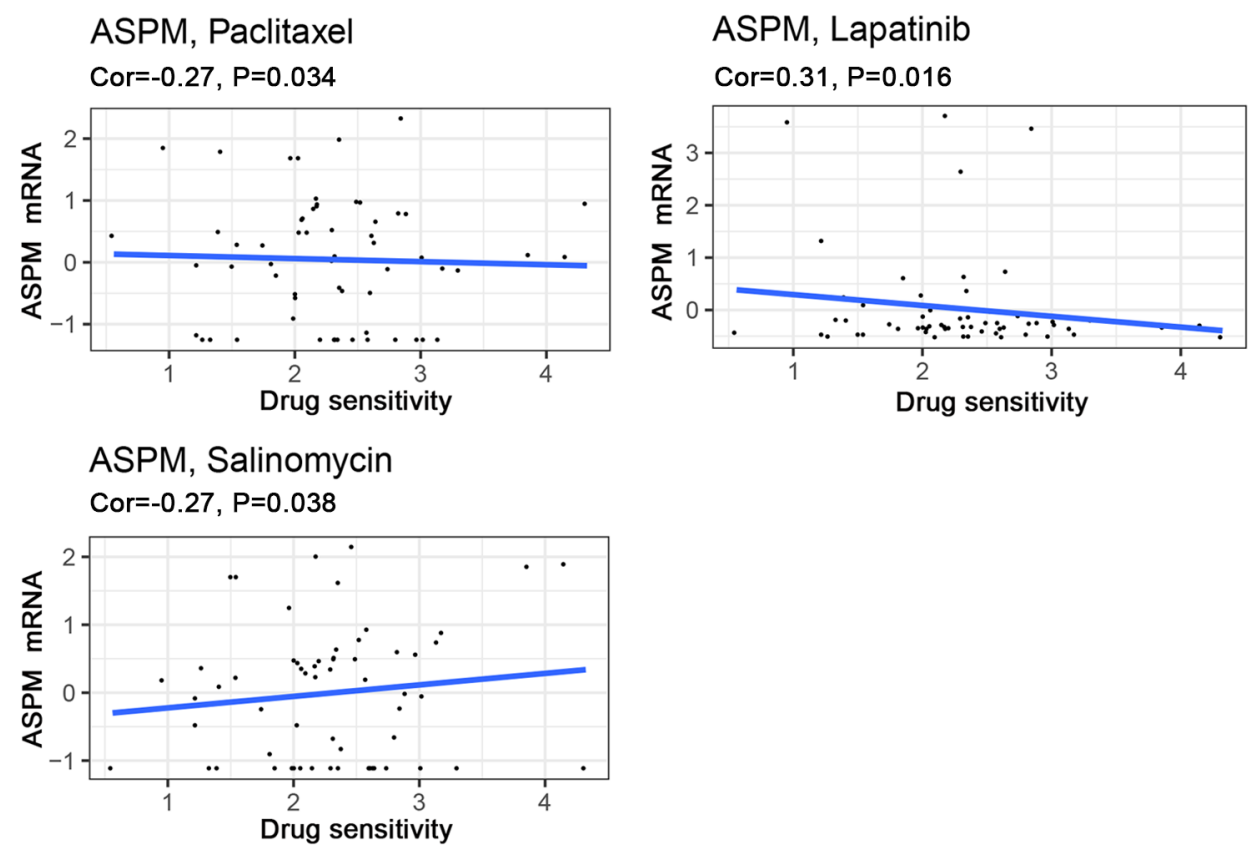

Figure S5 Correlations between anticancer drug sensitivity and the expression of ASPM based on CellMiner database. 